\title{
Experimental Simulation of Turbine-Exhaust Oxygen Recovery
}

\author{
Jim A. Clark ${ }^{*}$ and Ryan W. Brandt ${ }^{\dagger}$ \\ Pratt \& Whitney Space Propulsion, West Palm Beach, FL, 33410-9600
}

\begin{abstract}
In some liquid-propellant rocket engines, the liquid-oxygen boost pump is driven by a turbine that is powered by high-pressure gaseous oxygen. Once it exits the turbine, this gaseous oxygen can be salvaged by injecting it into the subcooled liquid oxygen exiting the boost pump. If the main LOX pump is to function correctly under these circumstances, complete condensation of the gaseous oxygen must quickly follow its injection into the boost-pump discharge. The current investigation uses steam and water in a simple rig that allows the condensation process to be visualized and quantified. This paper offers dimensionless-parameter correlations of the data and trends observed.
\end{abstract}

\section{Nomenclature}

$D C D=D$ imensionless $C$ ondensation $D$ istance $=$ condensation distance divided by vapor-port diameter

DiamRatio $=$ vapor-injection-port diameter divided by liquid-tube diameter

MomRatio = vapor-injection momentum (density times velocity) divided by liquid momentum

$T_{\mathrm{liq}} \quad=$ temperature of liquid before it reaches the vapor-injection port

$T_{\text {boil }} \quad=$ boiling temperature of the liquid at the pressure in the liquid tube, near the vapor-injection port

$T_{\text {mix }} \quad=$ enthalpy-based calculation of $T_{\text {liq }}$ after all vapor has been condensed

$T_{\text {mixratio }} \quad=T_{\text {mix }}$ (absolute temperature) divided by $T_{\text {boil }}$ (absolute temperature)

$T_{\text {thresh }} \quad=$ the value of $T_{\text {liq }}$ at which $\mathrm{DCD}=1.0$

$T_{\text {threshratio }}=\mathrm{T}_{\text {thresh }}$ (absolute temperature) divided by $\mathrm{T}_{\text {boil }}$ (absolute temperature)

\section{Introduction}

T liquid-propellant rocket engines, a boost pump is sometimes used to raise propellant pressure to a level that is 1 well above the propellant's saturation pressure yet well below the final pressure that is produced in the main pump. This arrangement allows the propellant-storage tank to operate at low pressure, it allows the boost pump to be optimized for suction performance, and it allows the main pump to be optimized for efficiency and packaging without compromise related to cavitation. To power a liquid-oxygen boost pump, a turbine driven by gaseous oxygen can be used, and the gaseous oxygen can be recovered by injecting it into the liquid oxygen emerging from the boost pump. The result of this injection is the condensation of the gaseous oxygen in the liquid oxygen being discharged from the boost pump.

In the condensation-based recovery of gaseous propellant in a boost turbopump, it is vital that the condensation process be complete before the liquid stream enters the main pump. The persistence of vapor bubbles in the liquid stream at the main-pump entrance can adversely affect main-pump performance. To quantify the distance needed to completely condense turbine-exhaust gas under various operating conditions, the current investigation attempted to experimentally simulate turbine exhaust-gas recovery by condensation in a flowing liquid stream. In the current

\footnotetext{
"Manager, Aerothermal Group, MS 713-31.
}

* Engineer, Aerothermal Group, MS 713-31. 
investigation, steam was a simulant for the turbine-exhaust gas, and liquid water was a simulant for the liquid propellant that is discharged from a boost pump.

\section{Previous Research}

Florschuetz and Chao ${ }^{1}$ examined the collapse rates of individual spherical vapor bubbles either under the influence of gravity or free of the influence of gravity in a quiescent pool of liquid. They described such collapse as being controlled by pressure differential (liquid pressure minus vapor pressure) and/or by heat transfer, and they formulated dimensionless collapse times to describe the process. Wittke and $\mathrm{Chao}^{2}$ analyzed the effect of bulk motion of the liquid on vapor-bubble collapse times, finding that the times depended on the Jakob number (sensible heat divided by latent heat of condensation) and Peclet number (the product of Reynolds number and Prandtl number, a combination often used to correlate forced-convection heat-transfer data). Hao and Prosperetti ${ }^{3}$ suggested that the heat transfer associated with vapor-bubble collapse is enhanced by fluid motion at the liquid-vapor interface. Levenspiel ${ }^{4}$ studied the heat transfer associated with the condensation of steam bubbles in subcooled water, reporting that bubble diameter, vapor density, and heat of vaporization were all influential variables.

The current investigation differed from these predecessors in several ways. First, the current experimental study had high liquid Reynolds numbers that are associated with turbulent flow. Second, the current work was focused on a continuous-flow vapor stream entering perpendicularly to a flowing liquid stream; the behavior of individual vapor bubbles was not discernible. Third, since individual bubbles could not be examined, the current investigation measured complete-condensation distances rather than bubble-collapse times.

\section{Experimental Apparatus}

For safety and availability reasons, saturated steam and liquid water were used in the current experimental study. Figure 1 is a sketch of the test rig. Warm water in a reservoir was circulated by a two-stage, constant-speed centrifugal pump through a 0.375 " ID tubing circuit that began and ended at the reservoir. Liquid flowrate through the circuit was controlled by a valve in the bypass loop, which allowed some pump-discharge water to return to the pump inlet. Downstream of the pump, a turbine flowmeter measured liquid flowrate, a thermocouple measured water temperature prior to the water's mixing with steam, and a transducer measured pressure in the mixing section. The steam-injection section of the circuit was a custom-made tee, such that the steam entered perpendicularly to the flowing water, whose path through the tee was straight. Clear polycarbonate tubing downstream of the tee allowed visualization and measurement of the location of the condensation-process termination, which was marked by an abrupt change in the transparency of the liquid stream. In other words, wherever the liquid contained vapor bubbles, it was cloudy, and wherever the vapor bubbles had all condensed, the liquid was clear. Downstream of the transparent tubing, a valve was available to control pressure in the mixing section. Finally, the liquid water, whose temperature had been increased by the injected steam, was returned to the reservoir. Reservoir temperature was controlled by continuous addition of cool water to the reservoir. This addition produced a continuous discharge of warm reservoir water through an overflow tube. Upstream of the mixing tee, saturated-steam flowrate was measured using the pressure drop across an orifice that had been previously calibrated with saturated steam.

In the current tests, liquid-water flowrates ranged from 6 to 18 gallons per minute, liquid-water temperatures ranged from $70 \mathrm{~F}$ to $195 \mathrm{~F}$, steam flowrates ranged from 0.005 to $0.021 \mathrm{lbm}$ per second, and mixing-section pressures ranged from 27 to 46 psia. Although the water pump was capable of producing higher pressures, the steam-supply pressure was limited to 65 psia. Thus, operating the mixing section at pressures above 46 psia would have produced an unacceptably low steam flowrate. Throughout all tests described in this paper, the liquid tube inner diameter remained constant $\left(0.375^{\prime \prime}\right)$. Steam-injection-port diameter was either $0.250^{\prime \prime}$ or $0.125^{\prime \prime}$ in all tests.

\section{Discussion of Results}

During a typical test with water circulating and steam flowing, externally supplied cool-water flowrate into the reservoir was adjusted to allow a very slow rate of rise of water temperature entering the pump. At low water temperatures, the steam-condensation distance was too small to measure. As the water warmed, the distance for complete steam condensation increased, allowing steam-condensation distance, water temperature, water pressure, water flowrate, steam flowrate, steam pressure, and steam temperature to be recorded at each of several specific condensation distances. Once the water temperature had risen to the point where complete condensation required a 
distance longer than what could be measured through the clear polycarbonate tubing, the test was terminated, and the reservoir was emptied and refilled with cool water in preparation for another test with a different combination of steam and water flowrates.

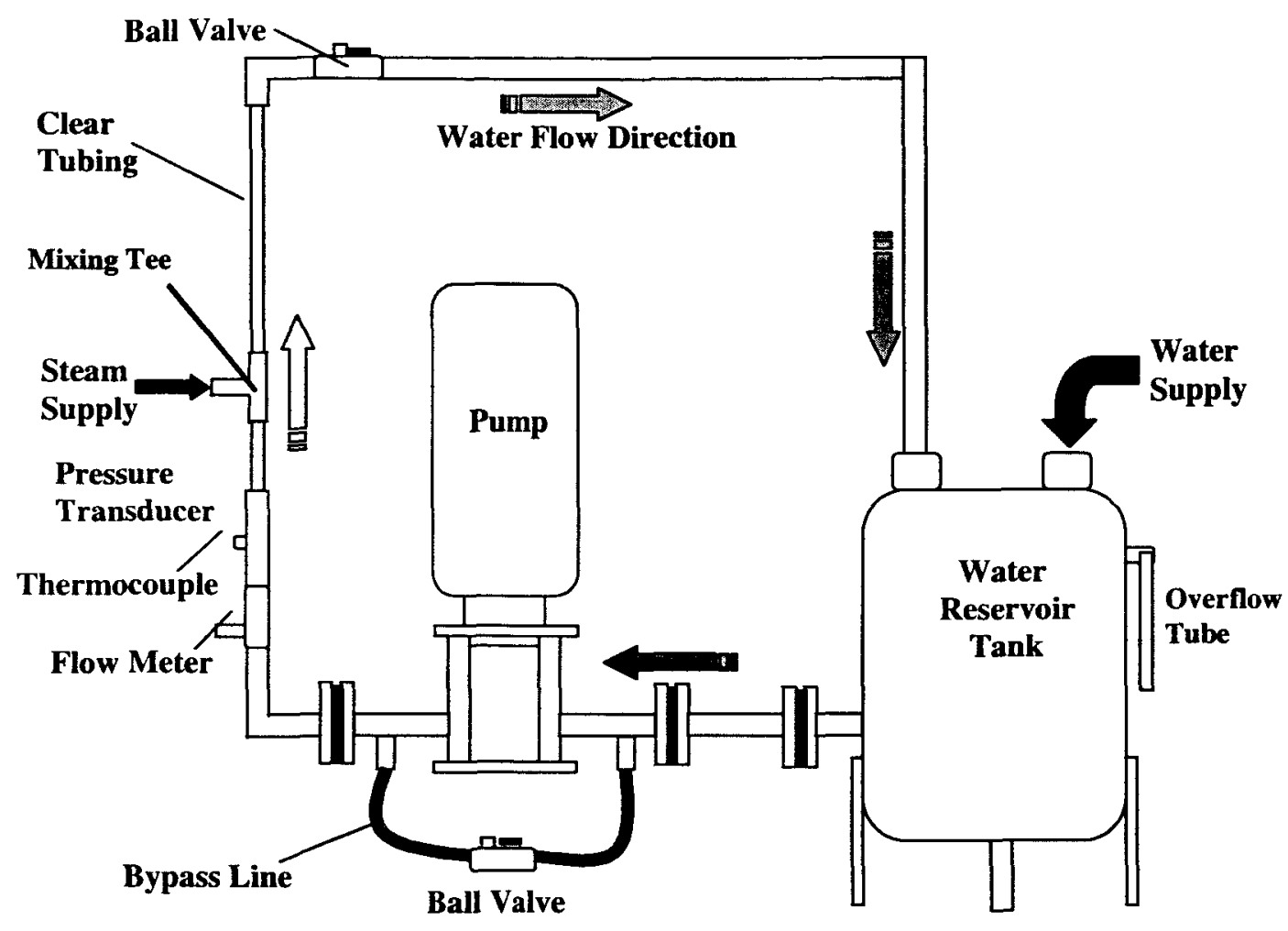

Figure 1. Schematic of Rig for Measuring Steam Condensation in Flowing Water

Figure 2 shows test data for a constant steam flowrate and a constant water flowrate. Steam-condensation distances have been normalized by steam-injection port diameter ( $\mathrm{DCD}=\mathrm{L} / \mathrm{D}$ ) because in general, the larger the steam jet, the longer the steam survives before the jet disintegrates and the condensation process is complete. Liquid temperatures (after mixing with steam) have been normalized by boiling-point temperature $\left(T_{\text {mixratio }}=T_{\text {mix }} / T_{\text {boil }}\right.$ ) to account for the observation that mixed-temperature proximity to boiling temperature is a better indicator of condensation performance than the liquid temperature alone. In presenting these data, Jakob number would have been a suitable substitute for $T_{\text {mixratio }}$ except that the traditional definition of Jakob number does not include the effects of vapor superheat and variable liquid specific heat. In contrast, $T_{\operatorname{mix}}$, the enthalpy-based calculated temperature of the liquid after all steam has been condensed, includes both effects. Otherwise, the difference between Jakob number and $T_{\text {mixratio }}$ is purely algebraic; as liquid water approaches its boiling temperature, $\mathrm{T}_{\text {mixratio }}$ approaches 1.0, and Jakob number approaches 0.0. Note in Figure 2 that an equation of the form

$$
\text { Dimensionless Condensation Distance }=\exp \left(\mathrm{Cl} *\left(\mathrm{~T}_{\text {mixratio }}-\mathrm{T}_{\text {threshratio }}\right)\right)
$$

fits the data well. In this figure, the value of $T_{\text {threshratio }}$ is 0.808 , implying that for this combination of steam and water flowrates, the condensation distance is negligibly small if $\mathrm{T}_{\operatorname{mix}}$ is less than or equal to $80 \%$ of the boiling temperature. This implication is consistent with the investigators' experience: when flowing water temperatures are relatively cool, steam condenses immediately. 


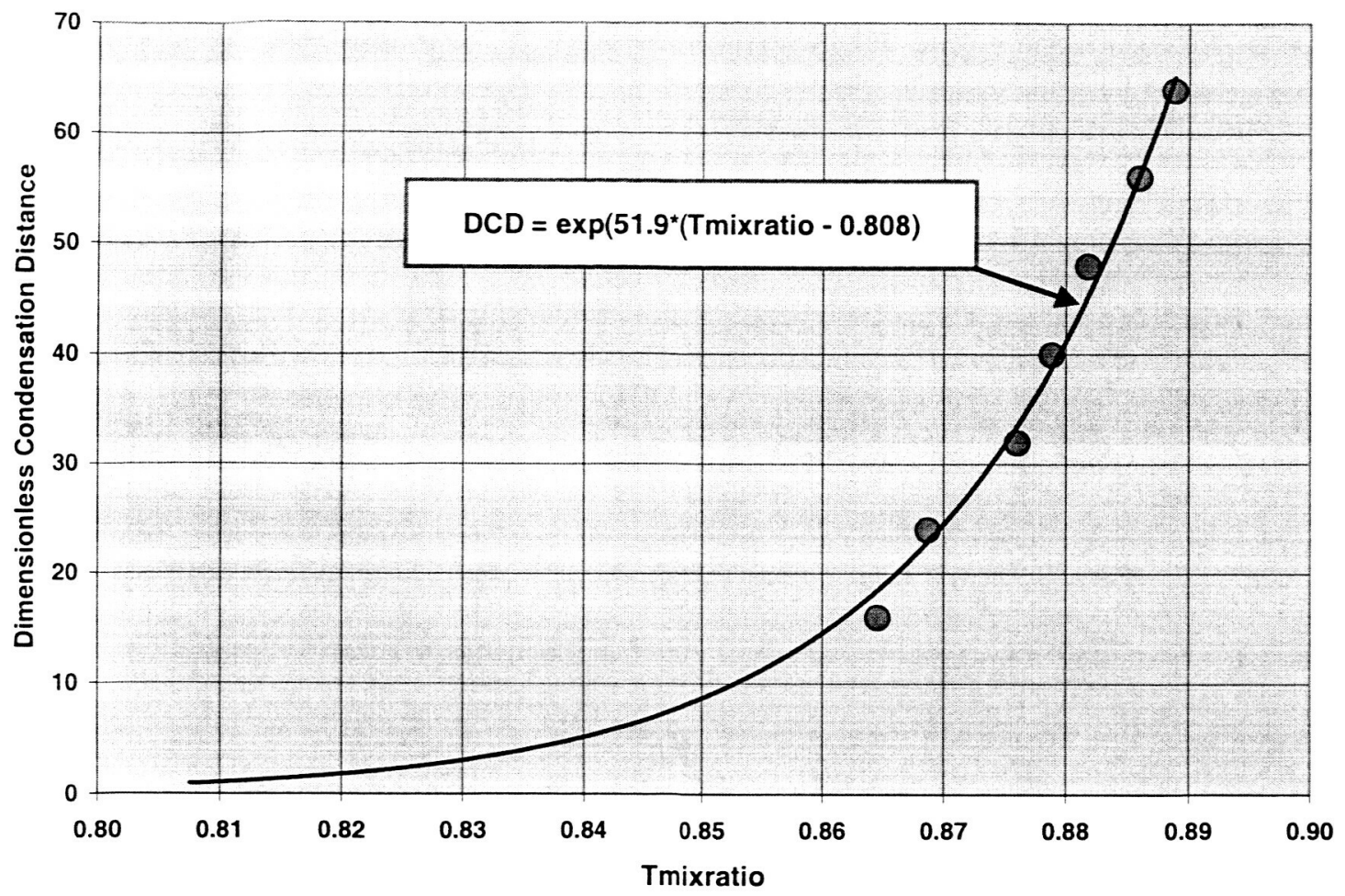

Figure 2. Dimensionless Condensation Distance vs. $\mathrm{T}_{\text {mixratio }}$ for DiamRatio $=0.33$, MomRatio $=0.77$

Figure 3 is a plot of all dimensionless condensation data for DiamRatio $=0.33$. Note in Fig. 3 that MomRatio ranged from 0.3 to 5 , and that the average value of $T_{\text {mixratio }}$ is higher when MomRatio is higher. High values of MomRatio promote rapid liquid-vapor mixing, which means that condensation can be quickly accomplished even when $\mathrm{T}_{\text {mix }}$ is near the boiling point. The trends of Fig. 3 indicate that dimensionless condensation data with MomRatio lower than 0.3 would fall only slightly to the left of the data that are already in Fig 3 . Similarly, Figure 3 indicates that dimensionless condensation data with MomRatio greater than 5 would fall only slightly to the right of the data that are already in Figure 3, because by definition, condensation cannot occur when $\mathrm{T}_{\text {mixratio }}$ is greater than or equal to one. Thus, for DiamRatio $=0.33$, it is reasonable to assume that there is an upper bound and a lower bound for the values of $\mathrm{T}_{\text {threshratio. }}$ Specifically, the $\mathrm{T}_{\text {threshratio }}$ lower bound appears to be close to 0.8 , and the $\mathrm{T}_{\text {threshratio }}$ upper bound appears to be close to 0.9. Therefore, an equation that appears to describe the influence of MomRatio on $T_{\text {threshratio }}$ is of the form,

$$
\mathrm{T}_{\text {threshratio }}=\mathrm{C} 2+\mathrm{C} 3 * \exp \left(-\mathrm{C} 4 / \mathrm{MomRatio}^{\mathrm{C} 5}\right)
$$

where $\mathrm{C} 2$ is the lower bound, and $(\mathrm{C} 2+\mathrm{C} 3)$ is the upper bound for $\mathrm{T}_{\text {threshratio, and } \mathrm{C} 5 \text { is an exponent larger than }}$ positive one. Figure 4 is a correlation (employing equations $(1,2)$ ) of all dimensionless-condensation data for DiamRatio $=0.33$.

Figure 5 is a plot of all dimensionless-condensation data for DiamRatio $=0.67$. Again, the higher the MomRatio,

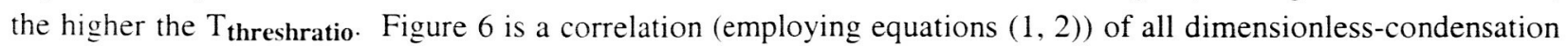
data for DiamRatio $=0.67$. 


\section{Effect of Mixed-Liquid-to-Boiling Temperature Ratio on Dimensionless Condensation Distance}

for Perpendicular Injection of Steam into Flowing Liquid Water.

Single-Port Steam Injection, DiamRatio $=0.33$

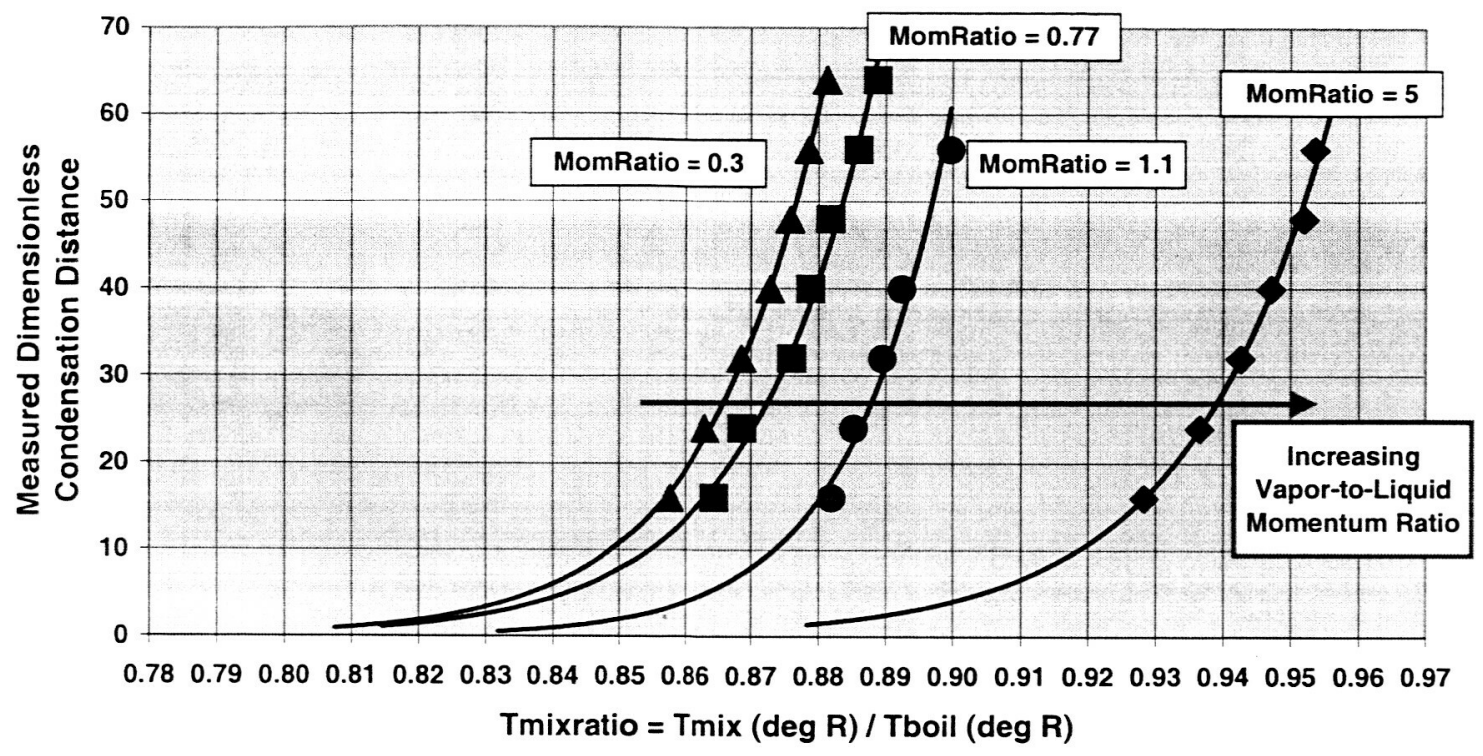

Figure 3. Dimensionless Condensation Distance vs. $\mathrm{T}_{\text {mixratio }}$ for DiamRatio $=0.33 ; 0.3<$ MomRatio $<5.6$

Single-Port Perpendicular Injection of Steam into Flowing Liquid Water

Correlation of Dimensionless Condensation Distances

DiamRatio $=0.33 \quad 0.3<$ MomRatio $<5.6$

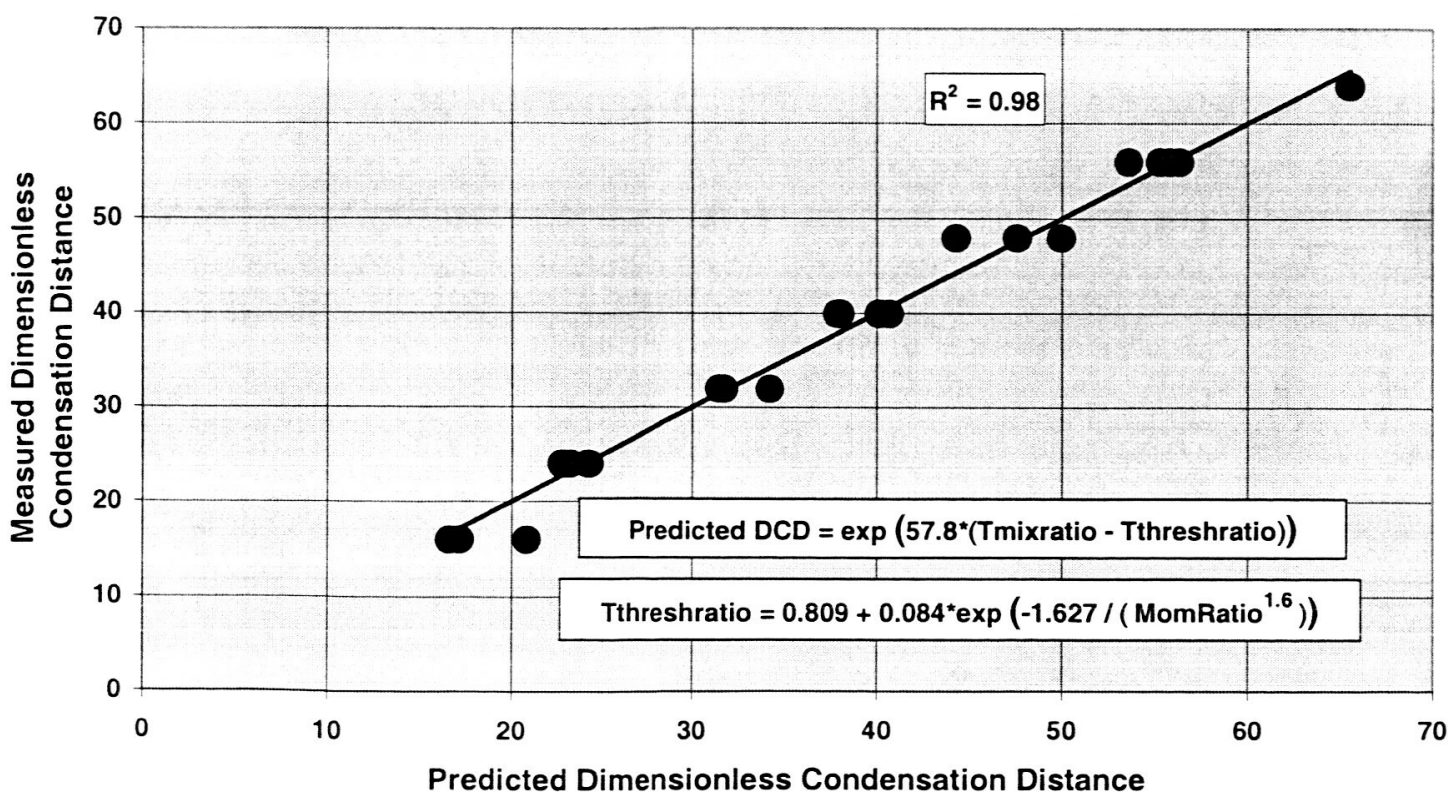

Figure 4. Correlation of Dimensionless Condensation Distances for DiamRatio $=0.33$ 


\section{Effect of Mixed-Liquid-to-Boiling Temperature Ratio}

on Dimensionless Condensation Distance

for Perpendicular Injection of Steam into Flowing Liquid Water

Single-Port Steam Injection, DiamRatio $=0.67$

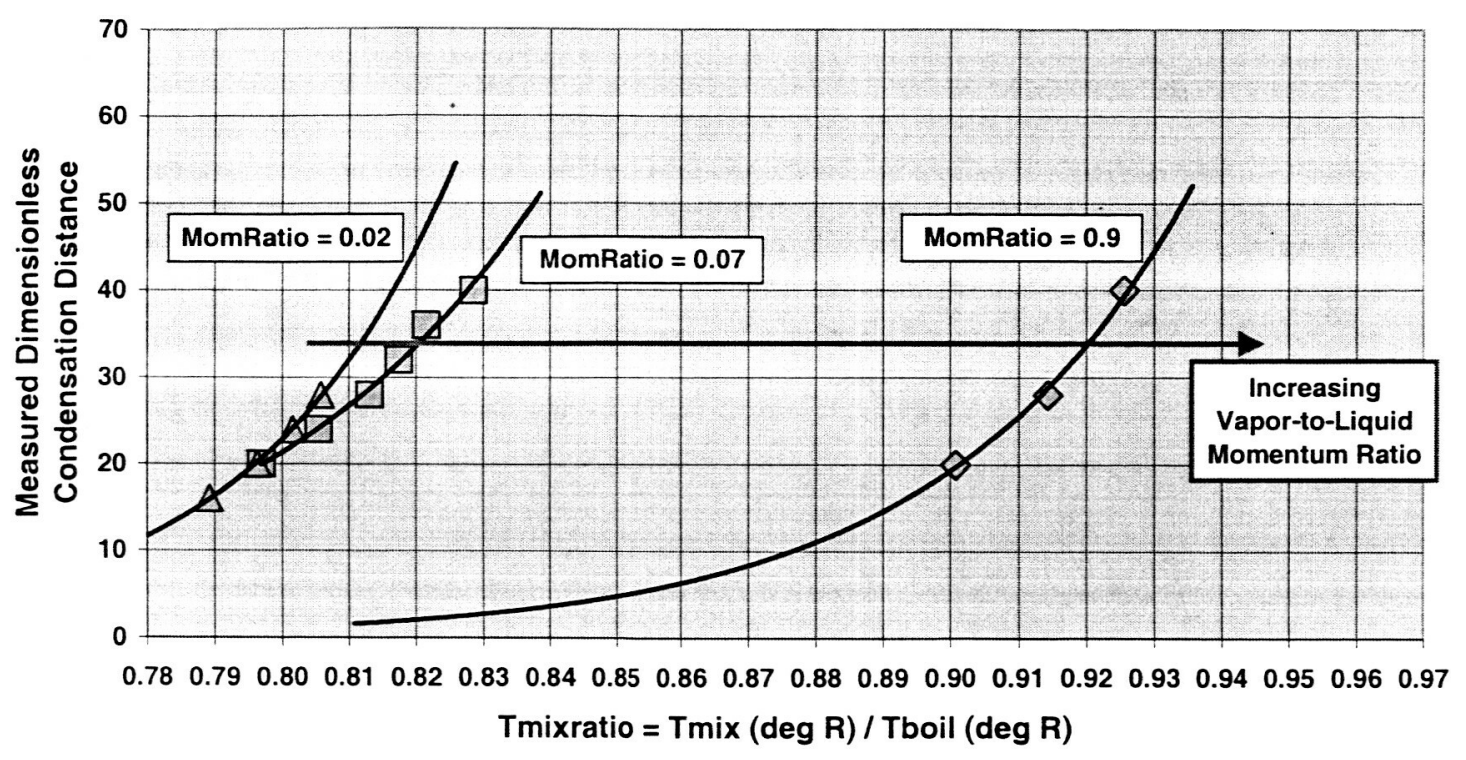

Figure 5. Dimensionless Condensation Distance vs. $\mathrm{T}_{\text {mixratio }}$ for DiamRatio $=0.67 ; 0.02<$ MomRatio $<0.9$

Single-Port Perpendicular Injection of Steam into Flowing Liquid Water Correlation of Dimensionless Condensation Distances

DiamRatio $=0.67 \quad 0.02<$ MomRatio $<0.9$

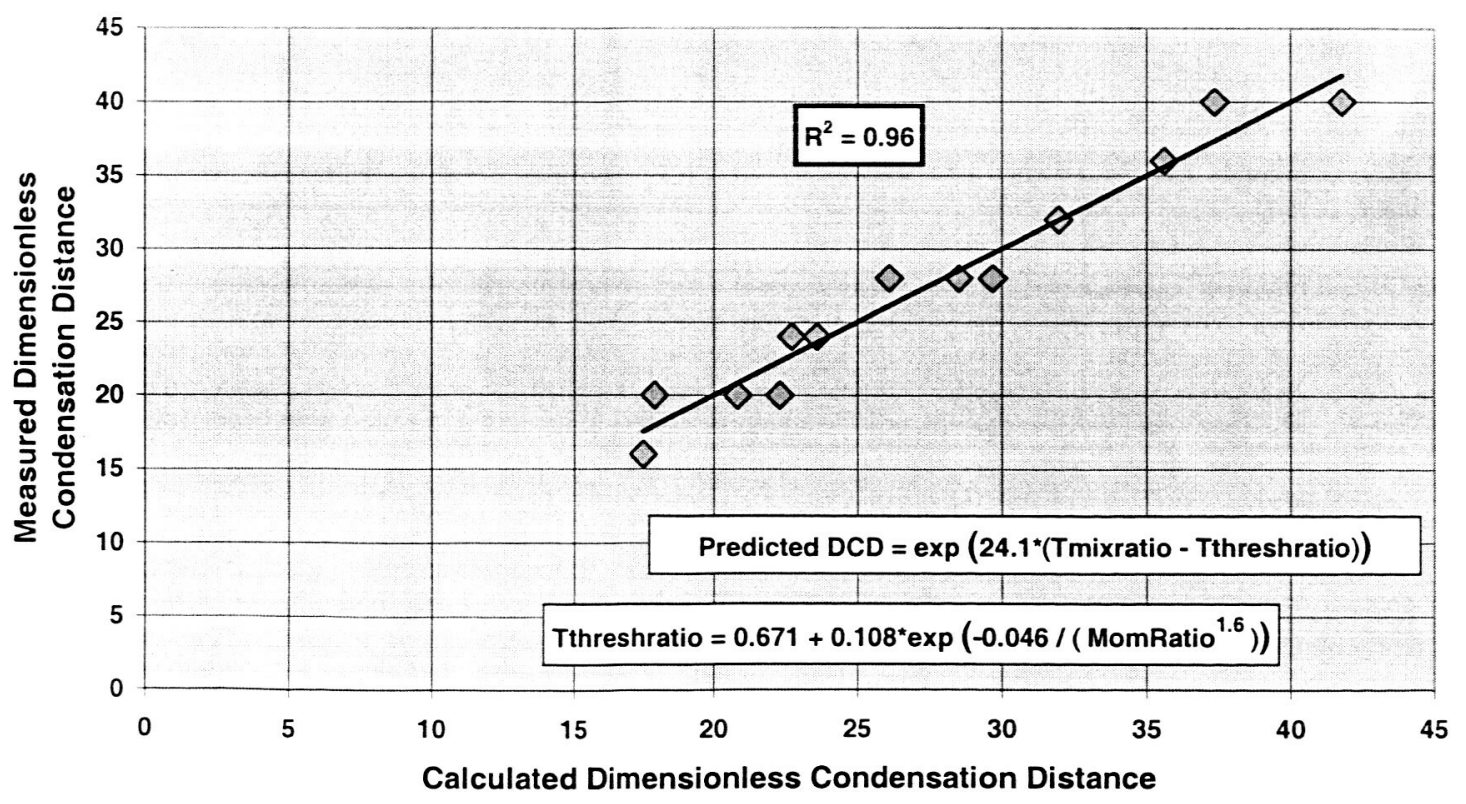

Figure 6. Correlation of Dimensionless Condensation Distances for DiamRatio $=0.67$ 


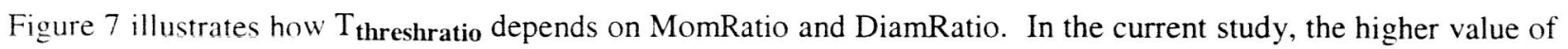

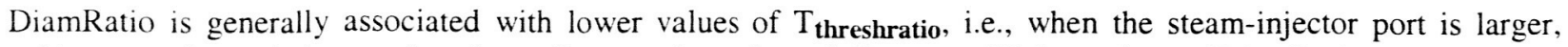
colder water is needed to produce immediate condensation of all steam. Higher values of MomRatio are associated

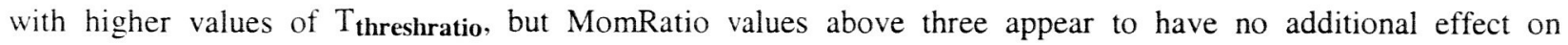

Threshratio.

\section{Single-Port Perpendicular Injection of Steam into Flowing Liquid Water Relationship Among MomRatio, DiamRatio and Tthreshratio}

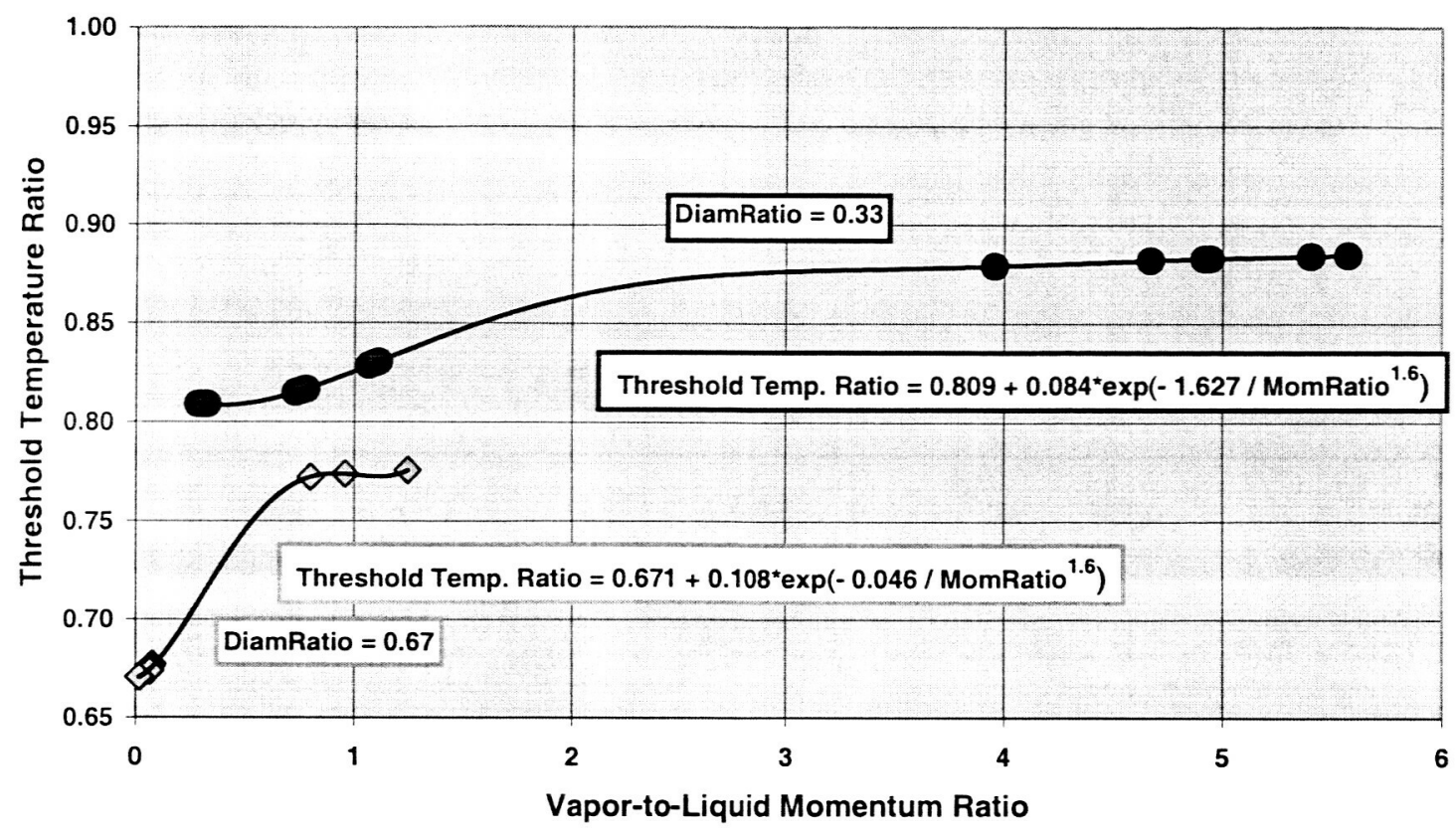

Figure 7. The Effect of MomRatio and DiamRatio on $\mathrm{T}_{\text {threshratio. }}$

Figure 7 suggests that a single equation involving MomRatio and DiamRatio might be used to predict $\mathrm{T}_{\text {threshratio. }}$. That equation could take the form

$\mathrm{T}_{\text {threshratio }}=(\mathrm{A} 1+\mathrm{A} 2 *$ DiamRatio $)+$

$$
\mathrm{A} 3 *(1-\exp (\mathrm{A} 4 * \text { DiamRatio })) * \exp \left((\mathrm{A} 5+\mathrm{A} 6 * \text { DiamRatio }) / \text { MomRatio }{ }^{\mathbf{1 . 6}}\right)
$$

where $\mathrm{C} 2$ in equation (2) has been replaced by $(\mathrm{A} 1+\mathrm{A} 2 * \mathrm{DiamRatio})$ in equation (3). Also, $\mathrm{C} 3$ in equation (2) has been replaced by $\mathrm{A} 3 *(1-\exp (\mathrm{A} 4 *$ DiamRatio)) in equation (3), and $\mathrm{C} 4$ in equation (2) has been replaced by (A5 +A6*DiamRatio) in equation (3). The forms of these substitutions were chosen to allow calculation of reasonable values of $\mathrm{T}_{\text {threshratio }}$ for Momratio from 0 to 6 and for DiamRatio from 0 to 0.67 .

After the same fashion, Figures 4, 6 suggest that a single equation involving DiamRatio, $\mathrm{T}_{\text {mixratio, }}$, and $\mathrm{T}_{\text {threshratio }}$ can be used to predict dimensionless condensation distance. That equation has the form

$$
\mathrm{DCD}=\exp \left((\mathrm{A} 7+\mathrm{A} 8 * \text { DiamRatio }) *\left(\mathrm{~T}_{\text {mixratio }}-\mathrm{T}_{\text {threshratio }}\right)\right.
$$

Figure 8 is the final overall correlation of all dimensionless condensation data using Equations $(3,4)$.

A legitimate criticism of the correlation in Figure 8 is that it relies on too many fitting constants to achieve a high correlation coefficient. The authors acknowledge this aspect of the correlation. However, in several ways, the form of the correlation is sensible. For example, in equation (4), the rate of rise of the exponential term would be expected to vary with DiamRatio, because vapor-jet diameter affects the rate of mixing, which affects condensation 
distance. Similarly, in equation (3), the minimum value of $T_{\text {threshratio, i.e., the value of } T_{\text {threshratio }} \text { when }}$ MomRatio is near zero, should reasonably be expected to depend on DiamRatio. When the vapor-jet diameter is larger, colder liquid is needed for instant condensation of all of the vapor. Finally, the magnitude of the effect of

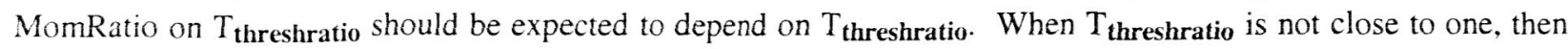

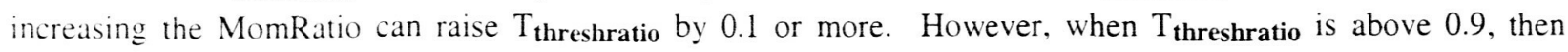

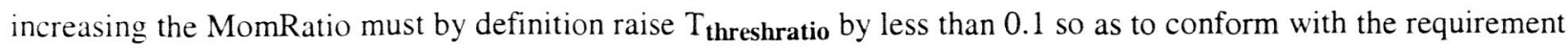

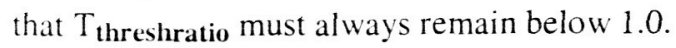

\section{Overall Correlation for Dimensionless Condensation Distance Single-Port Steam Injection Perpendicular to Flowing Water $0.33<$ DiamRatio < $0.67 \quad 0.02<$ MomRatio $<5.6$}

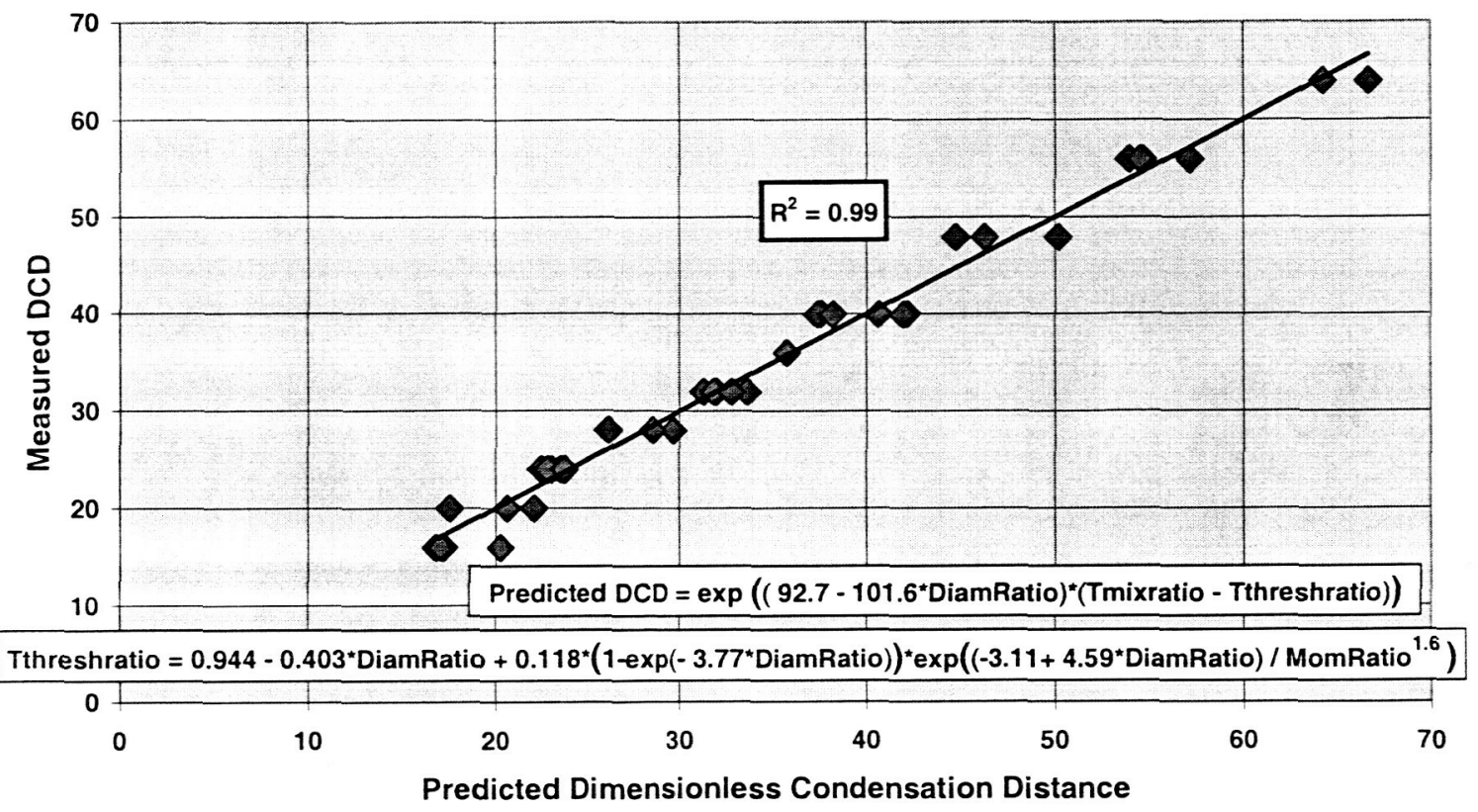

Figure 8. Overall Correlation for Dimensionless Condensation Distance of a Single Vapor Jet in Flowing Liquid.

Although equations $(3,4)$ on Figure 8 produce reasonable values of DCD when DiamRatio is less than 0.33 , the extrapolation of equations $(3,4)$ using values of DiamRatio significantly above 0.67 is problematic. The equation (3) linear substitution for $\mathrm{C} 2$ in equation (2) indicates that the minimum value of $\mathrm{T}_{\text {threshratio }}$ should be equal to 0.54 when the DiamRatio equals one. However, the investigators' brief experimentation with a geometry involving

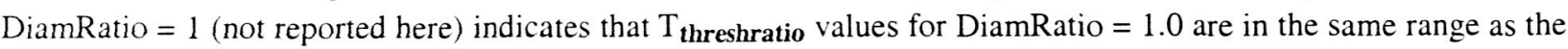
$\mathrm{T}_{\text {threshratio values for a DiamRatio }}=0.67$, probably because of the blockage presented by a steam jet whose diameter is equal to the liquid-stream diameter. In this situation, the liquid stream does not have the option of flowing around the vapor jet. Instead, the liquid stream is forced to penetrate and vigorously mix with the vapor jet. Thus, the extrapolation of equations $(3,4)$ for values of DiamRatio greater than 0.67 is not recommended, although such extrapolation may be safe for values of MomRatio less than 0.1 , i.e., when the vapor jet does not significantly block the liquid stream.

8

American Institute of Aeronautics and Astronautics 


\section{Conclusions}

This paper has presented dimensionless condensation distances for single-port, perpendicular, continuous injection of steam into turbulent, flowing liquid water. Condensation distances have been shown to depend on vapor-port diameter, MomRatio, DiamRatio, and $\mathrm{T}_{\text {mixratio }}$. As expected, the lower the value of $\mathrm{T}_{\text {mixratio }}$ and the smaller the vapor-port diameter, the shorter the condensation distance. Also, elevated values of MomRatio promote mixing, which shortens condensation distance.

The effect of DiamRatio is more complex. At low MomRatio (less than 0.5) mixing is minimized, and the condensation process is made less efficient by DiamRatio increases. At high MomRatio (greater than 0.5) a largerdiameter vapor jet's increased penetration of the liquid stream promotes mixing, thereby accelerating the condensation process.

Nearly instantaneous condensation appears achievable if the value of $T_{\text {mixratio }}$ is kept below 0.6 , regardless of MomRatio or DiamRatio. This finding indicates that the influences of vapor-injection geometry and momentum may be muted by the careful selection of boost-pump operating conditions. However, even at $T_{\text {mixratio }}$ values greater than 0.6 , instantaneous condensation can also be achieved with a small vapor port in combination with a MomRatio above two.

\section{Recommendations for Further Research}

To fully understand the physics of continuous-flow vapor condensation in a flowing liquid stream, more experimental work is needed. For single-port vapor injection, condensation distances need to be measured for DiamRatio $>0.67$, and for vapor-injection that is not perpendicular to the liquid stream. Furthermore, multi-port vapor injection should be explored. At first glance, multi-port vapor injection seems like a scheme that can minimize vapor-condensation distance, because the size of each vapor-injection port is smaller than it would be for a single port with the same total port area. However, at low MomRatio, multi-port vapor injection may worsen condensation performance because small vapor jets do not penetrate a liquid stream as far as a single jet of the same flowrate and MomRatio. In addition, the port-to-port spacing of a multi-port injection scheme may significantly impact the condensation process; vapor ports that are in line with one another or too close to one another may inhibit mixing between the liquid and the vapor. Finally, for condensation tests with either single-port or multi-port vapor injection, use of superheated vapor or a vapor-liquid combination other than steam-water is desirable, to learn whether the correlations developed in the current effort apply to such situations.

\section{Acknowledgments}

The National Aeronautics and Space Administration sponsored this work under contract \# NAS8-01108. The authors thank Brian Goode of NASA-MSFC for his consultation and advice during the test program. The authors also thank Charles Whipple of Pratt \& Whitney Space Propulsion for developing the steam-condensation rig described in this paper.

\section{References}

'Florschuetz, L. W. and Chao, B. T., "One the Mechanics of Vapor Bubble Collapse," Journal of Heat Transfer, May, 1965 , pp. $209-220$.

${ }^{2}$ Wittke, D. D. and Chao, B. T., "Collapse of Vapor Bubbles with Translatory Motion," Joumal of Heat Transfer, February, 1967, pp. $17-24$.

${ }^{3}$ Hao, Y. and Prosperetti, A., “The Collapse of Vapor Bubbles in a Spatially Non-uniform Flow," International Journal of Heat and Mass Transfer, Vol. 43, 2000, pp., 3539 - 3550.

${ }^{4}$ Levenspiel, O., "Collapse of Steam Bubbles in Water," Bubble Dynamics, Vol. 51, No. 6, 1959, pp., 787 - 790. 